\title{
Tolerance
}

\section{A virtue during times of stress}

\section{Kenneth Maiese}

Editor-in-Chief; Professor and Chair; Department of Neurology and Neurosciences; University of Medicine \& Dentistry of New Jersey; New Jersey Medical School; Newark, NJ USA

Environmental conditions that surround a cell or organism can be in rapid flux that can result in adverse outcomes. Through time, adaptive mechanisms have developed to cope with changes in the environment and ultimately protect against cell injury. In particular, cellular systems have become tolerant to injury to the extent that is defined by the words of Friedrich Neitzsche who stated "That which does not kill you makes you stronger." For example, during cardiac or nervous system ischemic injury, sublethal exposure to oxidative stress can lead to the induction of gene expression and translation of proteins that will later provide protection against future insults of oxidative stress. Furthermore, these protective pathways may even develop following significant injury to a cell and may offer future protection against different oxidative stress insults rather than the just the initiating insult. These results suggest that central pathways within a cell may be responsible for the determination of eventual tolerance against numerous environmental insults.

In this issue of Oxidative Medicine and Cellular Longevity, we offer a novel group of papers that present new work to examine the ability of a cell or an organism to endure detrimental changes in their surrounding environment. In their review paper, Essick and Sam evaluate the role of autophagy in several systems and disorders of the body and illustrate how under some circumstances autophagy is necessary for cell development, cell differentiation, and cell tolerance, such as during starvation. Yet, the pathways of autophagy under different environmental settings also may lead to the degradation of a cell and its ultimate demise. Setshedi et al. provide insight into the role of acetaldehyde and how it may lead under different circumstances to chronic alcoholic liver disease or hepatocellular carcinoma. There appear to be a number of factors that play a causative role but genetic components and their association to different environmental stimuli such as oxidative stress clearly have a strong impact upon the ability of a cell to tolerate the effects of acetyldehyde. In his review paper on anoxic tolerance, Storey brings together the role of constitutive as well as inducible antioxidant defenses that affect acute processes of injury, but he also argues for the significant pathways that impart protection for more long-term consequences associated with aging and cell longevity. Muir et al. offer insight into the clinical parameters that affect endothelial cell function in patients with coronary artery bypass grafting and demonstrate that gender, environmental toxins such as tobacco exposure, and metabolic parameters associated with diabetes may each contribute to the function and tolerance of the vascular system in patients with coronary artery disease. In the next paper, Atukeren et al. identify albumin as a pathway for tolerance in the body and show that loss of the antioxidant biological activity of albumin is associated with changes in its redox status. Raicevic et al. with their clinical study of women bearing a distressed fetus offer new insight into the role of serum superoxide dismutase/catalase levels in these patients as potential assessment of fetal distress and offer new considerations for these pathways of oxidative stress in relation to fetal and maternal responses to tolerate and prevent injury. In our final paper, Choi et al. heighten our awareness of the dual ability of pathways that may normally be responsible for tolerance against injury, but interestingly may be detrimental during other environmental conditions. The authors show that in a model of vitiligo that exposure to the toxin dopamine leads to redox-sensitive Akt activation, a pathway usually linked to cell protection, but under the experimental conditions examined, Akt was associated with cell death. The studies in this issue of Oxidative Medicine and Cellular Longevity clearly echo the words of Neitzsche that insults which do not initially kill you can prepare you for future events. However, to an even greater depth the novel work in these papers highlight the complexity of pathways associated with tolerance and demonstrate for us that the environment, genetics and intrinsic pathways all play a significant role that can lead to a variety of outcomes. 


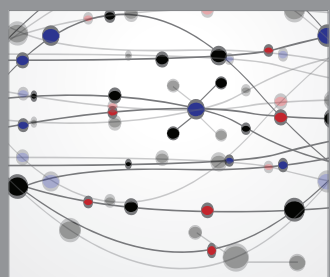

The Scientific World Journal
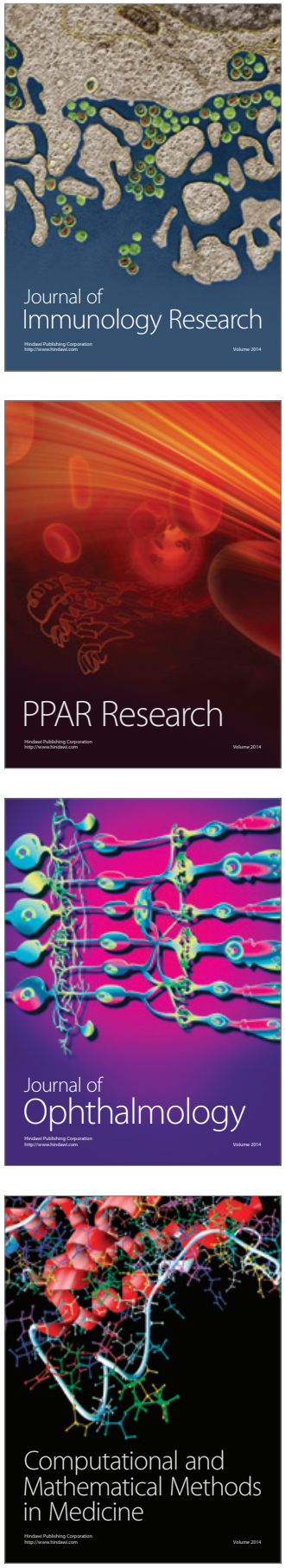

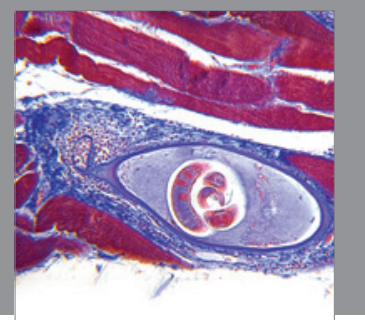

Gastroenterology

Research and Practice
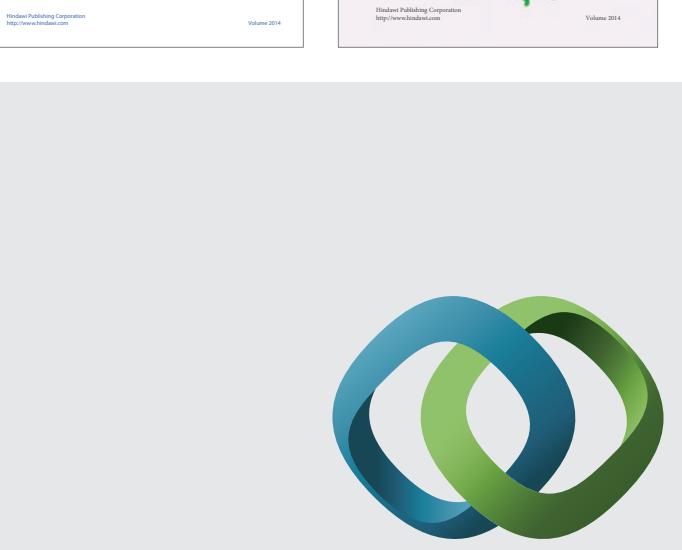

\section{Hindawi}

Submit your manuscripts at

http://www.hindawi.com
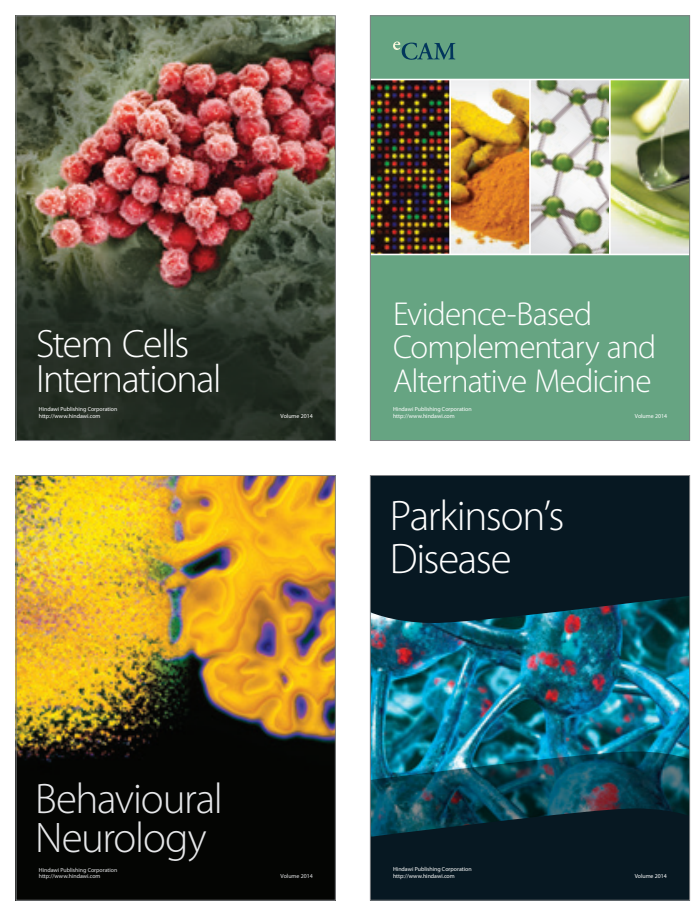

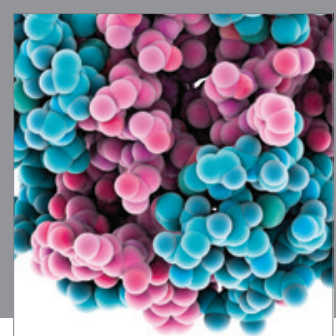

Journal of
Diabetes Research

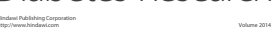

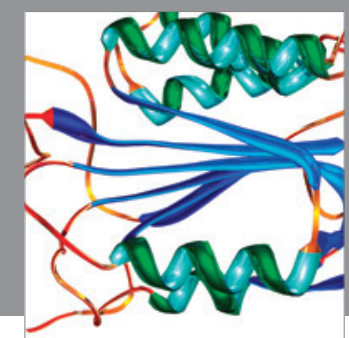

Disease Markers
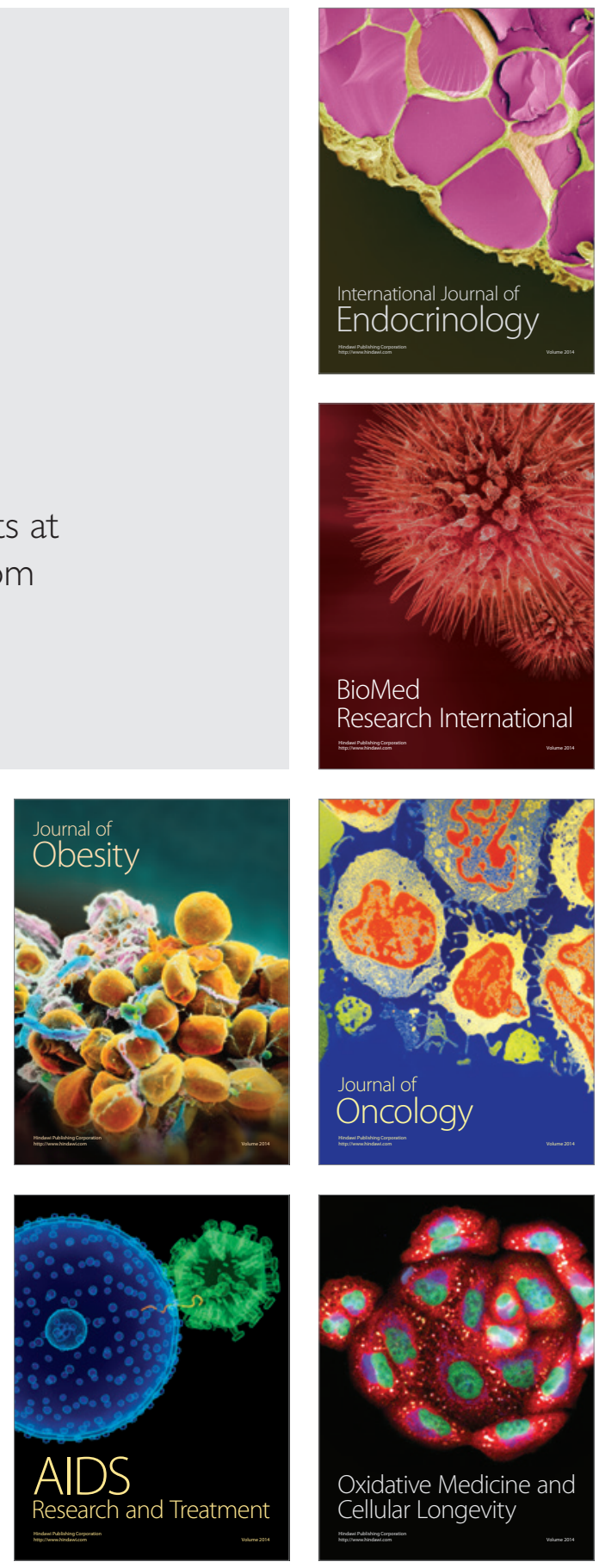